\title{
Charakterisierung von Zelladsorption auf Biomaterialien mittels Reflektometrischer Interferenzspektroskopie
}

\author{
J. Hutterer ${ }^{1}$ und G. Gauglitz ${ }^{1}$ \\ ${ }^{1}$ Institut für Physikalische und Theoretische Chemie, Eberhard-Karls Universität, Tübingen, Deutschland \\ Kontakt: johanna.hutterer@iptc.uni-tuebingen.de
}

\section{Einleitung}

Ob ein Implantat erfolgreich vom Körper des Patienten angenommen wird, hängt wesentlich von der Interaktion der umgebenden Zellen mit dem Material des Fremdkörpers ab. Die Stärke und Art der beteiligten Integrine, den Ankermolekülen der Zelle, an der Adhäsion wirkt sich auf die Migration, die Proliferation und Genexpression der adhärenten Zellen aus [1]. Eine Vielzahl an Oberflächeneigenschaften wie die chemische Struktur, die Rauigkeit und mögliche Vorbehandlungen beeinflussen den Adhäsionsprozess ebenso wie der Typ der beteiligten Zellen. Zudem unterscheiden sich die Anforderungen an eine Oberfläche in Bezug auf die Zell-Material Interaktion sehr stark je nach Implantat und Anwendung. Beispielsweise ist eine gute Osseointegration - initiiert durch das Anwachsen der körpereigenen Osteoprogenitorzellen - zentral für ein gutes Einwachsen von Knochenimplantaten [2], während ein Anlagern von Thrombozyten an Implantaten im Herzkreislaufsystem eine Hauptursache von Komplikationen darstellt [3]. In Anbetracht der komplexen Vorgänge an der Oberfläche während der Zelladhäsion auf Biomaterialen und der sehr unterschiedlichen Anforderungen an das spätere Verhalten der Zellen an der Oberfläche bedarf es eines fundierten Wissens über diese Vorgänge an der Grenzfläche zwischen lebendem Gewebe und künstlichem Implantat. Die zur Verfügung stehenden Methoden weisen jedoch Einschränkungen auf. Klassische Methoden wie die mikroskopische Untersuchung von adhärenten Zellen und Gewebeschnitte mit histologischen Färbungen oder Immunfärbung können meist nur Informationen über ZellMaterial-Interaktion am Ende des Versuches geben. Um die Anlagerung von Zellen an eine Oberfläche auch zeitaufgelöst untersuchen zu können bietet unter anderem die Interne Totalreflexionsfluoreszenzmikroskopie eine bessere Möglichkeit [4]. Da diese Methode jedoch auf den Einsatz von Markermolekülen angewiesen ist, entsteht zwangsläufig das Problem, dass nicht ausgeschlossen werden kann, dass die Markierungsreagenzien die natürlichen Vorgänge beeinflussen oder selbst mit der Zelle oder der Oberfläche interagieren. Aus diesem Grund sind vor allem direkte optische Methoden - wie „optical waveguide light mode spectroscopy" und Oberflächenplasmonenresonanzspektroskopie - für die Untersuchung von ZellMaterial-Interaktionen sehr vielversprechend. Diese Methoden, welche kleine Änderungen des Brechungsindex an einer Grenzfläche detektieren können, zeigen auch Limitierungen in Bezug auf die Eindringtiefe. Da diese Methoden auch eine starke Temperaturabhängigkeit aufweisen und von spezieller Oberflächenbeschaffenheit (Gold- film oder Gitter) abhängen, sind die Eisatzgebiete in Bezug auf die Untersuchung on Zell-Material-Interaktionen oft eingeschränkt.

Die Reflektometrische Interferenzspektroskopie (RIfS) [5] bietet ein hohes Potential einige dieser Probleme zu überwinden. Durch das direkte optische und zeitabhängige Funktionsprinzip verbindet es alle Vorteile der direkten optischen Methoden mit den Vorzügen der weitaus geringeren Temperaturabhängigkeit und einem linearen Zusammenhang zwischen angelagerter Schicht und dem Signal über Schichtdicken im Bereich mehrerer $\mu \mathrm{m}$. Mit einem integrieren Flusskanal können Zellösungen über die Oberflächen geführt und damit beispielsweise die fluiddynamischen Verhältnisse im Herzkreislaufsystem nachempfunden werden.

Im Zuge dieser Arbeit soll untersucht werden ob sich RIfS für Anwendungen zur Untersuchung von ZellMaterial-Interaktionen eignet. Hierfür werden als Modeloberflächen Glassubstrate, welche mit unterschiedlichen Proteinen der extrazellulären Matrix funktionalisiert wurden, untersucht. Die Beschichtung von Implantaten mit Proteinen, welche das Anwachsen der Zellen auf dem Implantat erleichtern sollen, ist gängige Praxis. Jedoch fehlt es auch hier an einem fundierten Wissen über die genauen Prozesse an der Oberfläche. Zudem wird erstmals untersucht ob die Bestimmung der kinetischen Ratenkonstanten aus den Bindungskurven der Zelladsorption möglich ist.

\section{Methoden und Materialien}

\section{Zellkultur}

Human Embryonic Kidney 293 (HEK) Zellen wurden in DMEM Medium (Sigma-Aldrich) versetzt mit $10 \%$ fetalem Kälberserum (Sigma-Aldrich), $1 \%$ L-Glutamin (SigmaAldrich) und $1 \%$ Penicillin/Streptomycin (Sigma-Aldrich) in T 25 oder T 75 Kulturflaschen kultiviert. Sobald die Zellen $80 \%$ Konfluenz erreicht haben wurden diese in einem Verhältnis 1:10 passagiert.

Bestimmung der Zellkonzentration: Sowohl für Herstellung der Zellsuspensionen für RIfS Messungen, die Untersuchungen der Zellkonzentration während der Messung, als auch für Adsorptionsexperimente auf Deckgläser für spätere Untersuchung der Morphologie und Zellzahl, wurden die Zellsuspensionen in der Neubauer Zählkammer unter einem Hellfeldmikroskop ausgezählt. Um tote von lebenden Zellen zu unterscheiden wurde die Zellensuspension 1:10 mit Trypanblau (Sigma-Aldrich), verdünnt. Der Anteil an toten Zellen in der Suspension wurde stets überprüft und durfte $5 \%$ nicht überschreiten. 
Herstellung der Zellsuspension: Nach dem Entfernen des Mediums aus der Kulturflasche und einem Waschschritt mit PBS (Thermo Fischer Scientific) wurden die Zellen mit Trypsin-EDTA (Gibco) von der Kulturflasche gelöst. Der Enzymverdau wurde nach circa 3 min mit der doppelten Menge Medium gestoppt, die Zellkonzentration bestimmt und nach Zentrifugation die sedimentierten Zellen in dem entsprechendem Volumen Medium gelöst.

Fixierung und Färbung der Zellen: Simultan zu den Adhäsionsversuchen in RIfS wurden jeweils 3 Deckgläser mit $450 \mu \mathrm{l}$ einer Lösung von $10 \mu \mathrm{g} / \mathrm{ml}$ Fibronektin, nativem Kollagen oder denaturiertem Kollagen für 10 min bei Raumtemperatur inkubiert und daraufhin mit PBS vorsichtig gewaschen. Nach einer weiteren Inkubation für $10 \mathrm{~min}$ mit $450 \mu \mathrm{l}$ Medium, wurde $300 \mu \mathrm{l}$ einer frisch hergestellte Zellsuspension von $10^{*} 10^{6} / \mathrm{ml}$ in Medium auf die Deckgläser gegeben. Nach einer Anlagerung der Zellen für $20 \mathrm{~min}$ unter stetem Schwenken oder in Ruhe wurden die Deckgläser mit Medium vorsichtig gewaschen und für weitere $15 \mathrm{~min}$ bei Raumtemperatur oder $120 \mathrm{~min}$ bei $37^{\circ} \mathrm{C}$ im Inkubator in Medium inkubiert. Die Deckgläser wurden vorsichtig mit PBS gewaschen und auf Objektträgern mit $65 \mu \mathrm{l}$ Paraformaldehyd (BioLegend) je Deckglas für $15 \mathrm{~min}$ behandelt. Nach einem weiteren vorsichtigen Waschschritt mit PBS wurden die Deckgläser mit $50 \mu \mathrm{l} 0.1 \%$ Triton $X$ (BioLegend) in PBS bedeckt, um die Zellmembrane für das Anfärben der Zellen zu öffnen. Nach einer Einwirkdauer von 15 min wurde dieses entfernt und die Deckgläser mit je $50 \mu \mathrm{l}$ FITC-Phalloidin (Sigma-Aldrich) Lösung 1:75 in PBS verdünnt auf die Deckgläser und für $45 \mathrm{~min}$ inkubiert. Daraufhin wurde die FITC-Phalloidin Lösung entfernt und $50 \mu \mathrm{L}$ DAPI Lösung (Sigma-Aldrich) 1:5000 verdünnt für 15 min auf die Deckgläser gegeben. Heraufhin wurden die Deckgläser dreimal vorsichtig mit PBS gewaschen und mit $5 \mu$ l ProLong ${ }^{\mathrm{TM}}$ Diamond Antifade Mountant (Thermo Fischer Scientific) mit der beschichteten Seite nach unten auf einen Objektträger geklebt. Am folgenden Tag würden die fixiert und angefärbten Zellen im Fluoreszenzmikroskop (Revolve, ECHO) untersucht.

\section{Reflektometrische Interfermzspektroskopie}

Messprinzip: Die Reflektometrische Interferenzspektroskopie ist eine direkte optische Methode um Vorgänge an einer Grenzfläche zu beobachten. Sie beruht auf der Interferenz von teilweisem reflektiertem weißen Licht an dünnen Schichten. Trifft Licht auf eine Grenzfläche zweier Stoffe mit unterschiedlichem Brechungsindex wird ein Teil des Lichtes transmittiert und ein Teil reflektiert (Abb. 1, A). Die reflektierten Teilstrahlen überlagern sich und das resultierende Interferenzspektrum wird vom Spektrometer aufgenommen. Für diese Arbeit wurde ein Interferenzglas hergestellt aus BK7 mit einer $10 \mathrm{~nm}$ dicken aufgedampften Schicht Tantalpentoxid gefolgt von einer weiteren Schicht Glas mit einer Dicke von $375 \mathrm{~nm}$ verwendet. Kommt es nun zu einer Anlagerung an die $375 \mathrm{~nm}$ dicke Interferenzschicht verändert sich der optische Weg des zweiten reflektierten Teilstrahl 12 und somit verschiebt sich das Interferenzspektrum (Abb. 1, B). Aus der Verschiebung der
Extrempunkte des Interverenzspektrums kann auf die optischen Schichtdicke nd geschlossen werden. Wird die Änderung der $\Delta$ nd über die Zeit aufgenommen spricht man von Bindungskurven (Abb. 1, C)
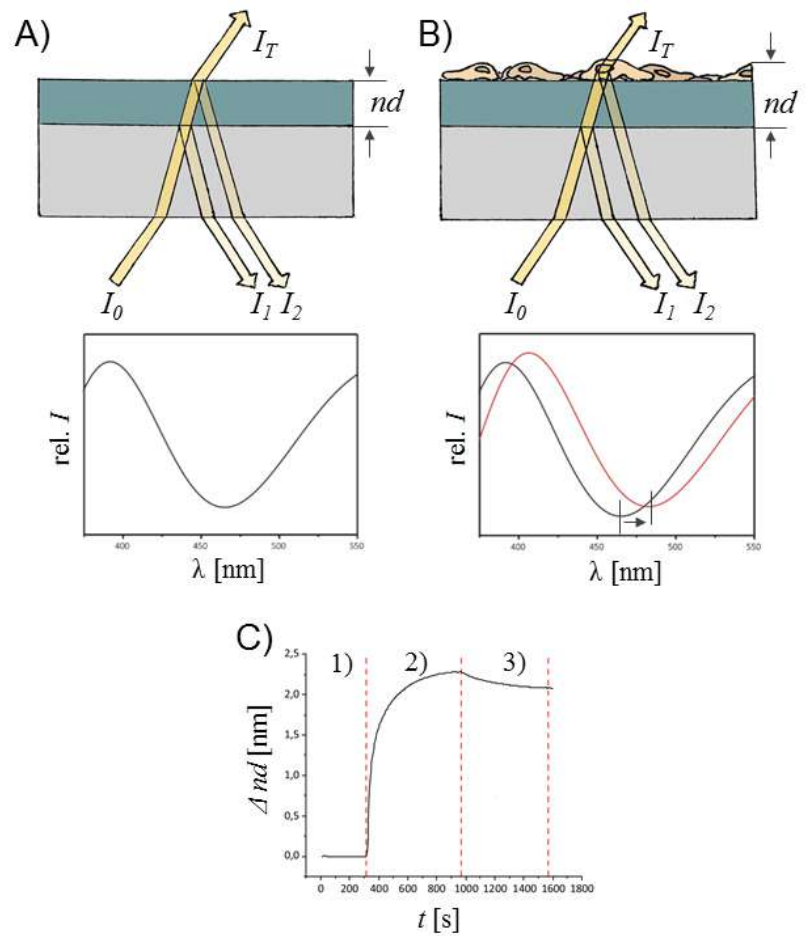

Abb. 1: Messprinzip der Reflektometrischen Interfernzspektroskopie.

Aufbau der RIfS Anlage: Als Weislichtquelle wurde eine Hallogenlampe verwendet (5 V, 10 W Halogenlampe, Osram) und mittels eines Spiegels, Linsen und Positionieroptik (Spindler \& Hoyer) auf den Lichtwellenleiter (Poly (methyl methacrylate), $1 \mathrm{~mm}$ Durchmesser, 50\%/50\% YFaserkoppler, Laser Components) fokusiert. Um zu vermeiden, dass schädliche UV-Strahlung und Wärmestrahlung mit den Zellen interagiert wurde ein Bandpass Filter (Spindler \& Hoyer) benutzt, um nur das Licht im erwünschten Spektralbereich passieren zu lassen. Über einen Arm des Y-Lichtwellenleiters wurde das Licht senkrecht von unten auf das Interferenzglas eingestrahlt. Das reflektierte Licht wurde über den zweiten Arm des Lichtwellenleiters zum Spektrometer (Diodenarrayspektrometer, SPEKOL 1100, Analytik Jena AG) geleitet. Um die Adsorptionsprozesse der Proteine und Zellen an der Oberfläche untersuchen zu können wurde alle $5 \mathrm{~s}$ ein Spektrum aufgenommen und $\Delta$ nd berechnet. Die jeweilige Probe wurde mit einer Peristaltikpumpe (Ismatec) durch dünne Schläuche und die Flusszelle über die Interferenzglasoberseite gepumpt. Die Flussrate betrug generell $0.5 \mu \mathrm{l} / \mathrm{s}$. Der Weg zwischen Probe und Flusszelle über den Schlauch wurde so kurz wie möglich gehalten. Um eine mögliche Schädigung der Zellen durch die mechanische Belastung durch die Peristaltikpumpe zu vermeiden wurde diese nach der Flusszelle in das System eingebaut. Da Zellen schon nach sehr kurzer Zeit sedimentieren wurde die Zellsuspension 


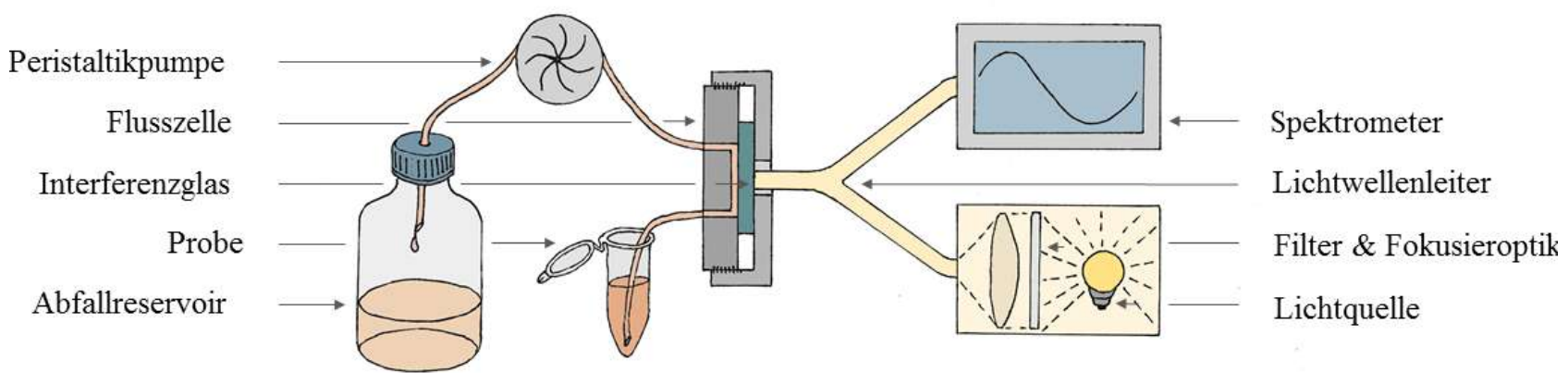

Abb. 2: Komponenten und Aufbau der Reflektometrischen Interfernzspektroskopie Anlage für die Untersuchung von Adsoptionsprozessen von Zellen.

über die gesamte Messdauer mit einem kleinen Rührfisch und Magnetrührer gemischt.

IfS Messungen: Für jede Messung wurde ein mit $\mathrm{KOH}$ und Piranha Lösung gereinigtes Interferenzglas benutzt. Zuerst wurde eine Baseline aufgenommen indem PBS über die Sensoroberfläche geleitet wurde. Im nächsten Schritt wurde für 10 min die Proteinlosung - Fibronektin (human, Sigma-Aldrich), natives Kollagen (human, SigmaAldrich) oder bei $75^{\circ} \mathrm{C} 10$ min denaturiertes Kollagen - zur Funktionalisierung der Sensoroberfläche über diese geleitet (Abb.3). Die Konzentration belief sich bei allen Messungen auf $10 \mu \mathrm{g} / \mathrm{ml}$.

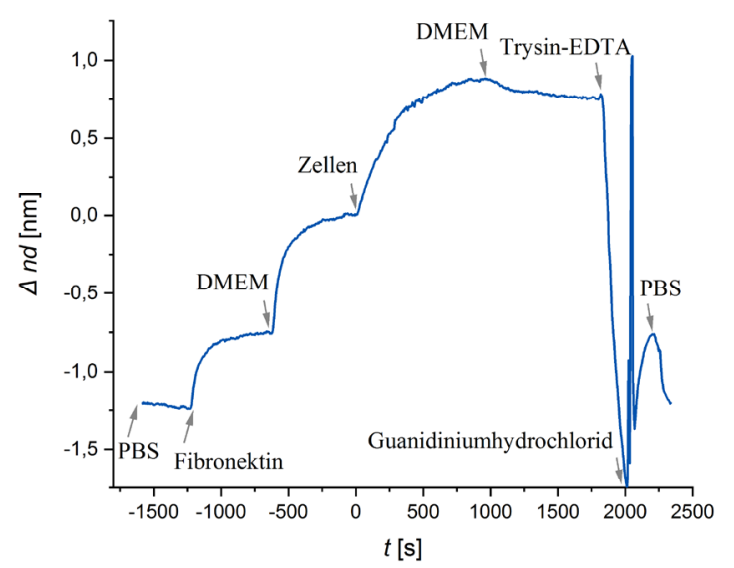

Abb. 3: Exemplarische Messung der Adsorption von $10 \cdot 10^{6}$ HEK-Zellen in Medium auf eine mit Fibronektin funktionalisierte Oberfläche mittels der Reflektometrischen Interfernzspektroskopie

Im folgenden Schritt wurde das Medium über die Oberfläche geleitet um diese, die Flusszelle und die Schläuche zu blocken und um die Signaländerung verursacht von Bestandteilen des Mediums von der Signaländerung durch die Adsorption der Zellen unterscheiden zu können. Dieser Schritt wurde für 10-17 min durchgeführt. Daraufhin wurde die Zellsuspension über die Oberfläche geleitet. Je nachdem wie viele Zellen zur Verfügung standen konnte dieser Schritt zwischen 10-20 min durchgeführt werden. Die Zellsuspension wurde jeweils frisch direkt vor der Injektion hergestellt. Die Dissoziation der Zellen wurde beo- bachtet indem erneut Medium über die Oberfläche geleitet wurde. Abgeschlossen wurde eine Messung mit der Reinigung des Interferenzglases, der Schläuche und der Flusszelle mit zuerst Trypsin-EDTA für mindestens $7 \mathrm{~min}$ um die Zellen wieder abzulösen und gegebenenfalls zu verdauen. Mit einer $1 \mathrm{M}$ Guanidiniumhydrochlorid Lösung wurden verbleibende Zellreste, Proteine und andere adsorbierte Stoffe für mindestens $7 \mathrm{~min}$ aus dem System entfernt. Bevor eine weitere Messung durchgeführt werden konnte wurde die Anlage für mindestens $10 \mathrm{~min}$ mit PBS gespült.

\section{Ergebnisse und Diskussion}

In Vorversuchen wurde zuerst getestet, ob eine Untersuchung der Zelladsorption auf Oberflächen mittels RIfS möglich ist. Hier wurde vor allem darauf geachtet, ob die Zellen den Versuch überleben können. Da weder Temperatur noch der $\mathrm{CO}_{2}$ Gehalt in der Luft während des Versuches kontrolliert wurde, wurden die Zellen nach einem RIfS Versuch mit Zellen, welche bei gleichen Bedingungen gelagert wurden und mit Zellen, welche im Inkubator gelagert wurden, untersucht. Nach der Bestimmung des Anteils an toten Zellen in der Zellsuspension wurde festgestellt, dass es keinen signifikanten Unterschied zwischen den Behandlungen der HEK-Zellen gab. Hieraus wurde geschlossen, dass weder die Scherkräfte, die Lichteinwirkung noch die Umgebungsbedingungen einen starken negativen Effekt auf die Zellen haben. Im nächsten Schritt wurde untersucht, ob die Anlagerung der Zellen im RIfS-Aufbau einen messbaren Unterschied in Messsignal zeigt. Da sich der Brechungsindex des Mediums nur wenig von dem der Zellen unterscheidet, wurde vermutet, dass möglicherweise nur sehr wenig Licht an der Phasengrenze zwischen Zelle und Medium reflektiert wird. Dies würde zu einer sehr kleinen Signaländerung während der Messung führen. Wie in Abbildung $3 \mathrm{zu}$ erkennen wurde $\Delta n d$ von circa $1 \mathrm{~nm}$ auf einer mit Fibronektin funktionalisierten Oberfläche durch die Anlagerung von $10 \cdot 10^{6}$ HEKZellen/ml in Medium gemessen. Das Signal zu Rauschverhältnis ist bei einem Signalzuwachs in diesem Bereich hinreichend gut. 
Im Folgenden wurde untersucht, ob mit Hilfe von RIfS unterschiedliches Adsorptionsverhalten von HEK-Zellen auf unterschiedlich funktionalisierten Oberflächen beobachtet werden kann. Funktionalisiert wurden die Oberflächen mit Fibronektin, nativem Kollagen oder denaturiertem Kollagen. Die Anlagerung der HEK-Zellen ist auf der mit denaturiertem Kollagen funktionalisierten Oberfläche am stärksten ausgeprägt. Hier werden Beladungen der Oberfläche von bis zu 2,6 nm verzeichnet während bei der mit nativem Kollagen und Fibronektin funktionalisierten Oberfläche $\Delta n d$ etwa $0,8 \mathrm{~nm}$ beträgt (Abb. $4, \mathrm{Abb}$. 5). Auch die Dissoziationsphase ist auf der mit denaturiertem Kollagen funktionalisierten Oberfläche am deutlichsten zu erkennen. In der Dissoziationsphase unterscheidet sich auch das Verhalten der HEK-Zellen auf den Fibronektin und Kollagen nativ funktionalisierten Oberflächen. Auf den Oberflächen mit nativem Kollagen haften die HEK-Zellen auch beim Spülen mit Medium stärker im Vergleich zu den beiden anderen getesteten Oberflächen.

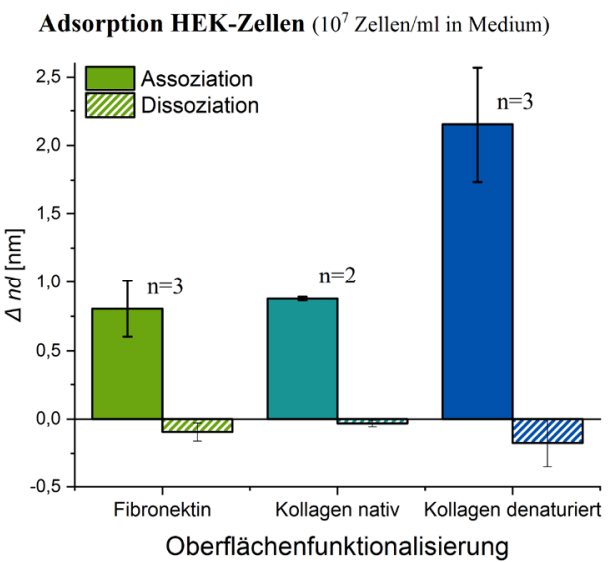

Abb. 4: Änderung der optischen Schichtdicke $\Delta$ nd durch die Assoziation und die Dissoziation von HEK-Zellen auf unterschiedlichen Oberflächen

Um zu beurteilen, wie sich die Zellmorphologie und die Anzahl der adhärenten Zellen im Vergleich zu den jeweiligen RIfS Messungen verhält, wurden auf Deckgläser adhärente Zellen fixiert und mit DAPI und FITC-Phalloidin angefärbt. Um die RIfS Messung möglichst gut auf einem Deckglas zu simulieren wurde die Zellsuspension währen der Anlagerung kontinuierlich leicht geschwenkt, um eine Sedimentation der Zellen auf die Oberfläche zu vermeiden. Nach einer Dissoziationsphase von 15 min wurden die Zellen fixiert und angefärbt (Abb. 6). Im Übersichtsbild ist klar zu erkennen, dass sich auf der mit denaturiertem Kollagen funktionalisierten Oberfläche etwas mehr Zellen befinden als auf den Oberflächen mit nativem Kollagen und Fibronektin. Auf beiden Kollagen Oberflächen agglomerieren die Zellen mehr als auf der Fibronektin Oberfläche. Im Cytoskelett der HEK-Zellen konnten nach der 15minütigen Dissoziationsphase keine Unterschiede zwischen den Oberflächen erkannt werden. Da der Fixierund Färbevorgang der Zellen mehrere Waschschritte beinhaltet, kann nicht davon ausgegangen werden, dass sich während dieses Prozesses keine Zellen von der Oberfläche gelöst haben. Dieser Einfluss kann auf unterschiedlichen Oberflächen unterschiedlich stark ausgeprägt sein. Deshalb können diese Ergebnisse nicht direkt verwendet werden, um eine mögliche Korrelation zwischen der Zelldichte auf der Oberfläche und der in RIfS gemessenen $\Delta n d \mathrm{zu}$ untersuchen. Jedoch zeigt sich ein gleicher Trend in beiden Verfahren was für eine Korrelation spricht. Zukünftig könnten die Interferenzplättchen direkt nach der Messung in der RIfS-Anlage unter einem Lichtmikroskop untersucht und danach fixiert und angefärbt werden.

Zur Untersuchung, wie sich die Zellen nach längerer Zeit auf der Oberfläche verhalten, wurde der Versuch mit einer längeren Dissoziationsphase von 120 im Inkubator wiederholt. In diesem Versuch wurde auf das Schwenken während der Assoziation verzichtet. Es befinden sich am Ende des Versuches deutlich mehr Zellen auf den Oberflächen (Abb. 7). Dies zeigt, dass die Scherkräfte einen sehr starken Einfluss darauf haben wie viele Zellen an der Oberfläche adhärieren können. Um vergleichbarere Bedingungen mit den RIfS-Messungen zu erreichen, ist es sinnvoll auch diese Versuche in einem Durchflusssystem mit definierten Fließgeschwindigkeiten durchzuführen. In den Übersichtsbildern nach der verlängerten Dissoziationszeit ist erkennbar, dass sich die meisten Zellen auf der mit Fibronektin funktionalisierten Oberfläche befinden. Die kleinste Zellzahl kann auf der Oberfläche mit denaturiertem Kollagen festgestellt werden, wobei die Oberfläche mit nativen Kollagen, im Vergleich zu den beiden anderen Oberflächen, eine mittlere Zellzahl aufweist.
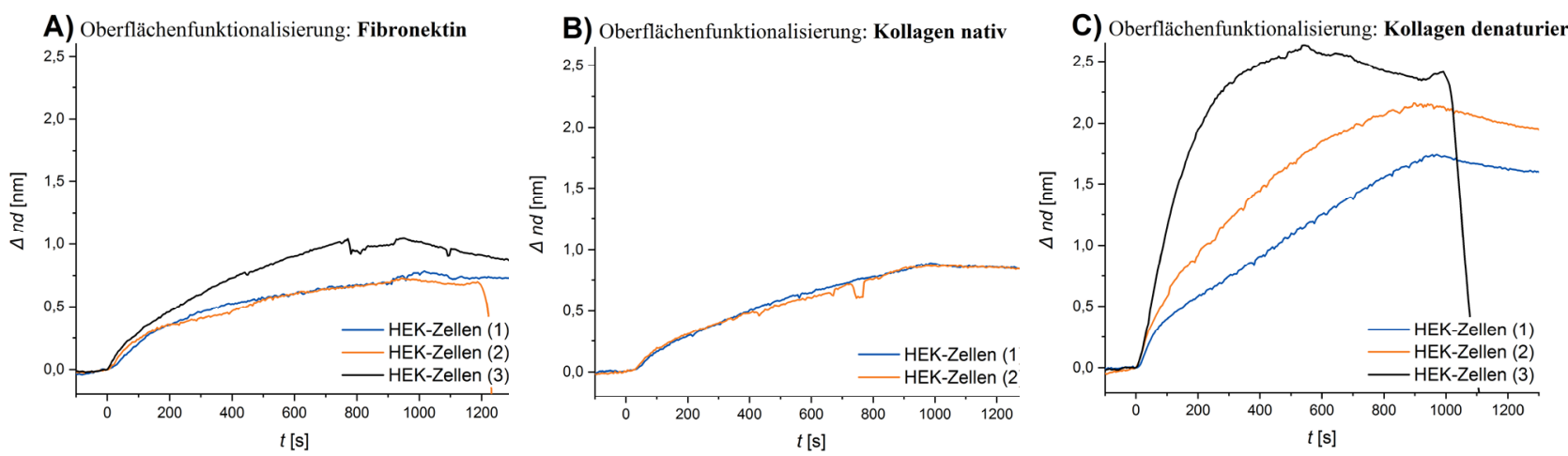

Abb. 5: Bindungskurven von $10 \cdot 10^{6}$ HEK-Zellen in Medium auf eine mit Fibronektin (A) eine mit nativem Kollagen (B) und eine mit denaturiertem Kollagen (C) (jeweils $10 \mu \mathrm{g} / \mathrm{ml}$ ) funktionalisierten Glasoberfläche. 

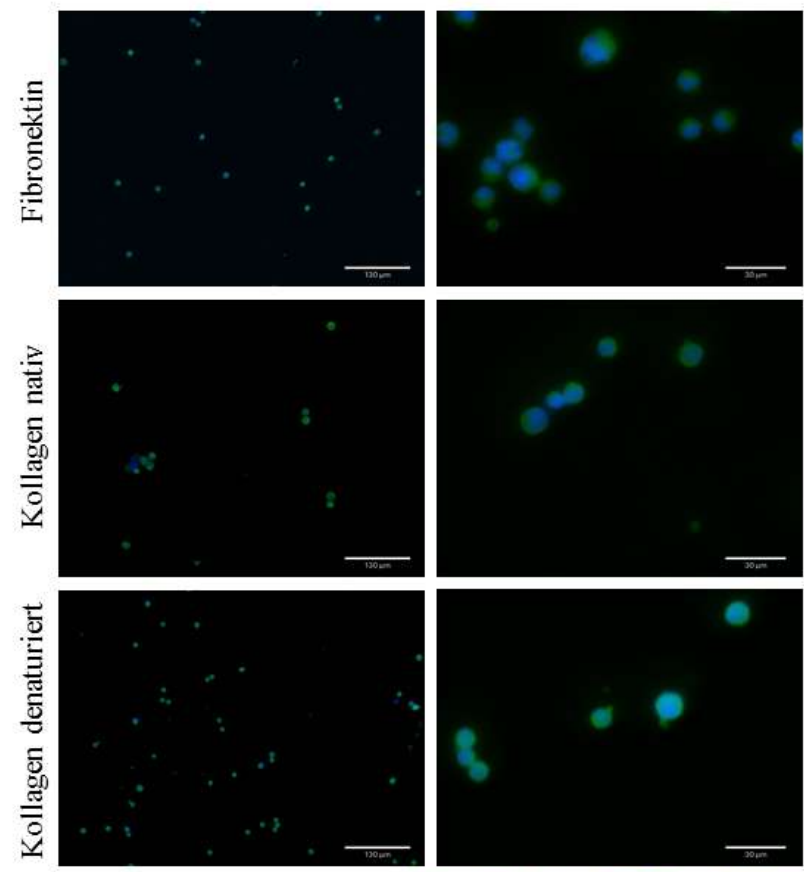

Phalloidin-FITC DAPI

Abb. 6: Fluoreszenzaufnahmen von HEK-Zellen auf unterschiedlichen Oberflächen. Assoziation: 15min RT unter Schwenken, Dissoziation: 15 min RT
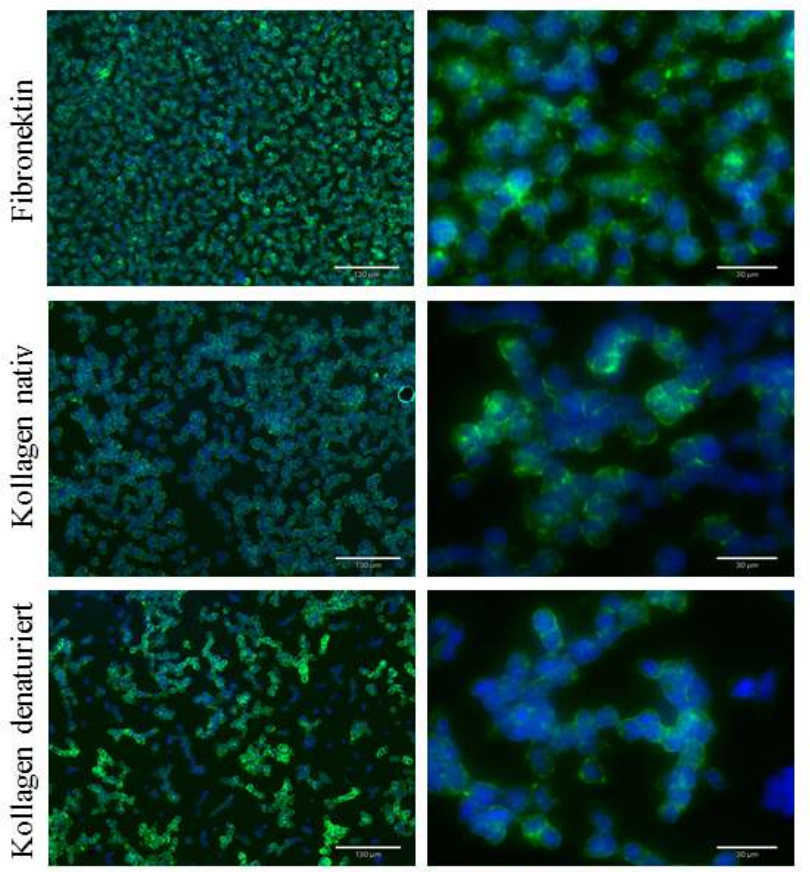

Phalloidin-FITC DAPI

Abb. 7: Fluoreszenzaufnahmen von HEK-Zellen auf unterschiedlichen Oberflächen. Assoziation: 15 min RT,

Dissoziation: 120 min in Inkubator

Die Fluoreszenzaufnahmen zeigen auch hier, dass die HEK-Zellen auf den Kollagen funktionalisierten Oberflächen stärker agglomerieren als auf der Fibronektin funktionalisierten Oberfläche. Zudem liegen die HEK-Zellen auf den Kollagen Oberflächen noch sehr abgerundet vor, während sie auf der Fibronektin Oberfläche deutlich spreiten.
Da gezeigt werden sollte, ob es auch möglich ist, die Anlagerungen von Zellen über einen längeren Zeitraum hinweg mit Hilfe von RIfS aufzuzeichnen, wurde die Dissoziationsphase in einem Versuch mit denaturiertem Kollagen funktionalisierten Oberflächen deutlich verlängert. Hierfür wurden die adhärierten Zellen für $3 \mathrm{~h}$ nach der Assoziation mit Medium gespült (Abb. 8). Hier ist deutlich zu erkennen, dass $\Delta n d$ zu Beginn der Dissoziationsphase deutlich fällt und dann eine kontinuierliche langsame Abnahme aufzeigt. Dieses Ergebnis zeigt, dass sowohl die Anfangsphase der Zelladsorption wie auch die weiteren Entwicklungen über mehrere Stunden mit diesem RIfS-Aufbau beobachtete werden können. In Vorversuchen wurde auch festgestellt, dass die $\Delta$ nd nach der Assoziation von HEKZellen auf einer Fibronektin Oberfläche bei Spülen mit Medium über mehrere Stunden langsam anwächst. Da die verwendeten Zellen aus einer anderen Charge stammten sind die Ergebnisse der nicht direkt mit der Langzeitmessung auf denaturiertem Kollagen vergleichbar, liefern aber einen Hinweis darauf, dass sich das Verhalten der HEKZellen auch in einem längeren Zeithorizont auf unterschiedlichen Oberflächen unterscheidet.

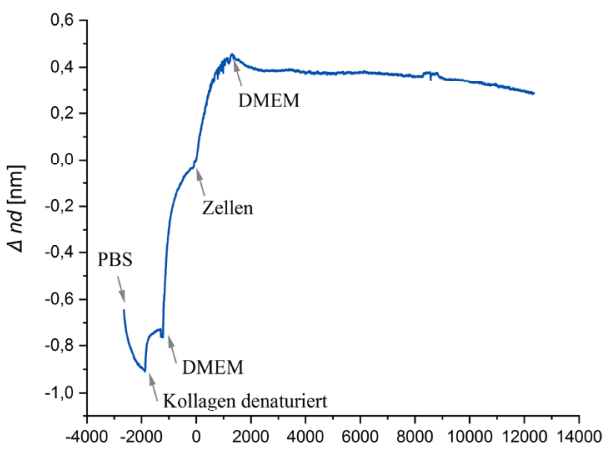

$t[\mathrm{~s}]$

Abb. 8: Langzeituntersuchung der Adsorption von $10 \cdot 10^{6}$ HEK-Zellen in Medium auf eine mit denaturiertem Kollagen funktionalisierte Glasoberfläche.

Obwohl kurze Zeit nach der Zelladsorption auf der Kollagen-funktionalisiert (denaturiert) Oberflächen im Vergleich zur Fibronektin-funktionalisierten Oberfläche mehr Zellen zu finden sind, befindet sich auf der KollergenOberfläche nach längerer Dissoziationsphase weniger Zellen. Dies ist ein Indiz, dass die Zellen stärker und spezifischer an Fibronektin im Vergleich zu Kollagen binden können.

Um detailliertere Aussagen zum Assoziations- und Dissoziationsverhalten und damit der Affinität von Zellen gegenüber einer Oberfläche treffen zu können, ist die Beschreibung dieses Vorgangs mit Ratenkonstanten anstrebenswert. In dieser Arbeit wurde erstmals getestet ob eine kinetische Auswertung der Adsorptionsprozesse von Zellen möglich ist. Eine Bedingung für die meisten Adsorptionsmodelle ist das Vorliegen einer Reaktion pseudoerster Ordnung. Um dies zu gewährleisten wird die Konzentration eines Reaktionspartners über den Reaktionsverlauf konstant gehalten. Da sich die Anzahl der freien Bindungsstellen auf der Oberfläche zwangsläufig mit der Zeit 
ändert wird klassischerweise die Konzentration des Analyten - in diesem Fall die Zellkonzentration - konstant gehalten. Durch die Minimierung der Wegstrecke zwischen Probengefäß und Sensoroberfläche und dem kontinuierlichen Rühren der Zellsuspension während des Versuches wurde versucht diese Anforderung zu erfüllen. Um dies zu überprüfen, wurde die Zellkonzentration während der RIfS Messung untersucht. Hierfür wurden während der Adsorption von $10 \cdot 10^{6} \mathrm{HEK}$-Zellen/ml auf einer Fibronektin Oberfläche alle 2 min eine Fraktion nach der Peristaltikpumpe entnommen und die Zellen in der Lösung ausgezählt. Die Ergebnisse zeigen, dass sich nach etwa 5 min eine Zellkonzentration ähnlich der theoretisch vorgegebenen Zellkonzentration einstellt. Im weiteren Verlauf schwanken die Werte stark um den theoretischen Wert (Abb. 9). Zudem ist die Zellkonzentrationsbestimmung über die Neubauer Zählkammer großer Streuung unterworfen. Mit einer hinreichenden Resuspension der HEK-Zellen unmittelbar vor der Messung könnte dieses Problem minimiert werden.

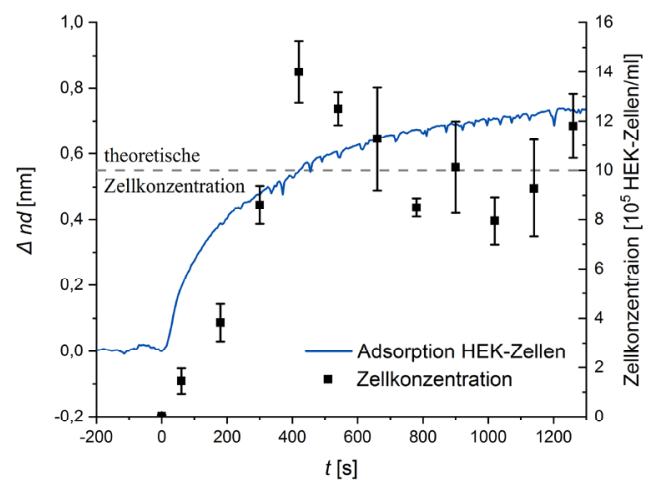

Abb. 9: Bestimmung der Zellkonzentration (Punkte und Fehlerbalken entsprechen Mittelwert und Standardabwei-

chung) während der Zelladsorption (blaue Linie).

Eine weitere Voraussetzung für die meisten Adsorptionsmodelle ist eine Abhängigkeit der Gleichgewichtsbeladung von der Konzentration des Analyten. Um dies zu testen wurde die Adsorption von $1 \cdot 10^{6} \mathrm{HEK}$-Zellen/ml, 5.10 HEK-Zellen/ml und $10 \cdot 10^{6} \mathrm{HEK}-Z e l l e n / \mathrm{ml}$ in Medium auf eine mit denaturiertem Kollagen funktionalisierten Oberflächen vermessen (Abb. 10).

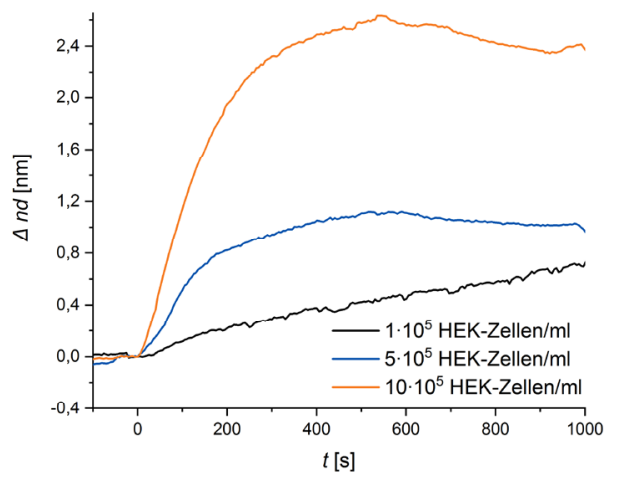

Abb. 9: Anlagerung von unterschiedlichen Konzentrationen an HEK-Zellen auf eine mit denaturiertem Kollagen beschichtete Oberfläche
Die Ergebnisse zeigen, dass eine Konzentrationsabhängigkeit vorliegt, wodurch die Bestimmung kinetischer $\mathrm{Pa}$ rameter der Zelladsorption theoretisch möglich ist. Hier ist jedoch zu beachten, dass eine konstante Zellkonzentration über die Messdauer bewahrt werden muss. Zudem ist - besonders wegen der Zellgröße - wichtig, den Einfluss der Diffusion auf die Zelladsorption auszuschließen.

\section{Ausblick}

Es wurde gezeigt, dass sich die Messmethode RIfS hervorragend eignet, um Adsorptionsprozesse von Zellen auf Oberflächen zu untersuchen. Die zeitabhängige Untersuchung der Änderung der optischen Schichte $\Delta$ nd ist sowohl für Beobachtungen in der ersten Phase der Adsorption im Bereich von mehreren Minuten wie auch über längere Zeiträume von mehreren Stunden möglich. Es konnte zudem gezeigt werden, dass sich die Adsorptionskurven von HEK-Zellen auf Fibronektin, nativem und denaturiertem Kollagen unterscheiden. Erstmalige Überlegungen zur Bestimmung kinetischer Parameter der Zelladsorption wurden vorgestellt. Es wurde gezeigt, dass deren Bestimmung theoretisch möglich ist. Zukünftig könnten ausgewählte Prozesse in dem komplexen Feld der Fremdkörper Reaktion an Implantaten von der Aktivierung der Thrombozyten an Oberflächen durch adhärente Proteine bis hin zur Interaktion von Oberflächen mit Leukozyten, Fibroblasten und Epithelzellen untersucht werden.

\section{Literatur}

[1] STREULI, C. H.: Integrins as architects of cell behavior. In: at - Molecular Biology of the Cell 27 (2016), Nr. 19, S. 2885-2888

[2] AGARWAL, R.; GARCÍA, A. J.: Biomaterial strategies for engineering implants for enhanced osseointegration and bone repair. In: Advanced Drug Delivery Reviews (2015), Nr. 94, S. 53-62

[3] EMMERT, M. Y.; FIORETTA, E. S.; HOERSTRUP, S. $P$.: Translational Challenges in Cardiovascular Tissue Engineering. In: Journal of Cardiovascular Translational Research 10 (2017), Nr. 2, S. 139-149

[4] UHLIG, K.; WISCHERHOFF, E.; LUTZ, J.-F.; LASCHEWSKY, A.; JAEGER, M. S.; LANKENAU, A.; DUSCHL, C.: Monitoring cell detachment on PEGbased thermoresponsive surfaces using TIRF microscopy. In: Soft Matter 6 (2010), Nr. 17, S. 4262-4267

[5] GAUGLITZ, G.; BRECHT, A.; KRAUS, G.; MAHM, W..: Chemical and biochemical sensors based on interferometry at thin (multi-) layers. In: Sensors and Actuators B 11 (1993), Nr. 1, S. 21-27

\section{Danksagung}

Diese Arbeit wurde vom Ministerium für Wissenschaft Forschung und Kunst Baden-Württemberg gefördert. Besonderer Dank gilt dem Arbeitskreis Weber im Interfakultären Institut für Mikrobiologie und Infektionsmedizin und dem Arbeitskreis Gauglitz (IPTC) an der Universität Tübingen. 